with two recommended DMARDS Grade IV: single use of DMARDS including MTX less than $5 \mathrm{mg}$.

Results: There was no relationship between titers of Anti-CCP Ab and titers of RF. We found significant statistical correlation between anti-CCP antibody titers and inflammatory markers such as CRP and MMP-3. There was significant statistical correlation between CRP and MMP-3.

In terms of treatment intensity, strong intensity group showed high titer of anti-CCP $A b$ and CRP. Titer of RF and MMP-3 level did not have any relationship with the treatment intensity. In cases treated with biologics, anti-CCP Ab and CRP were significantly higher compared to non-biologic case group. In $80 \%$ of cases treated with biologics titer of anti-CCP was more than 200 units. However, non-biologic treatment was continued in more than $50 \%$ of cases with anti-CCP Ab higher than 200 units.

Conclusions: Even though we treated cases based on the severity of the symptoms of the patient and response in laboratory data, high anti-CCP Ab titers and CRP at the base line were most associated with the treatment intensity after 1 year. The results of our study suggest that the titer of anti-CCP Ab can be better a predictor of the treatment intensity than MMP-3 and RF.

Disclosure of Interest: None declared

DOI: 10.1136/annrheumdis-2017-eular.4989

\section{AB0248 THE IMPACT OF THERAPY ON ANTI-CARBAMYLATED PROTEIN ANTIBODY ISOTYPES AND SEROSTATUS IN PATIENTS WITH} EARLY RA TREATED WITH ABATACEPT AND METHOTREXATE

L. Trouw ${ }^{1}$, S. Connolly ${ }^{2}$, A. Johnsen ${ }^{2}$, J. Ye $^{2}$, M. Maldonado ${ }^{2}$, R. Toes ${ }^{1}$,

T. Huizinga ${ }^{1}{ }^{1}$ Leiden University Medical Center, Leiden, Netherlands,

${ }^{2}$ Bristol-Myers Squibb, Princeton, United States

Background: Maturation of autoantibody responses has been suggested to be a proxy for disease maturation. Autoantibody responses against post-translationally modified antigens are present in autoimmune diseases and antibodies directed against carbamylated proteins (anti-CarP antibodies) are a marker of RA. AntiCarP antibody analysis in patients with early RA offers the opportunity to estimate whether specific intervention during such early stages of autoantibody development may have an impact on the maturation of the anti-CarP antibody response.

Objectives: We assessed the relationship between changes in anti-CarP isotypes and rates of seroconverson to negative in patients with early RA.

Methods: In the Assessing Very Early Rheumatoid arthritis Treatment study (AVERT; NCT01142726), patients with early RA were treated with abatacept (ABA)+MTX, ABA monotherapy or MTX alone. ${ }^{1}$ Patients in AVERT were anticyclic citrullinated peptide-2 positive at baseline for study entry. ${ }^{1}$ In this post hoc analysis, concentrations of anti-CarP isotypes were measured using custom ELISAs. Anti-CarP ELISAs for immunoglobulin (Ig)G, IgM or IgA isotypes were performed in patient serum at baseline, and at Days 85 and 365 on treatment. Baseline levels of each anti-CarP antibody isotype and \% seropositivity were comparable across the three treatment arms. Adjusted mean change from baseline was calculated using a longitudinal repeated measures model.

Results: At baseline, 51.3, 42.5 and $29.3 \%$ of all patients with serum available in AVERT were positive for IgG, IgM (indicative of an ongoing immunoresponse) and IgA anti-CarP isotypes, respectively. Overall, approximatly $65 \%$ of patients were positive for at least one anti-CarP antibody isotype. Median \% change from baseline $(25 \%, 75 \%)$ for anti-CarP isotypes levels from baseline to Days 85 and 365 are shown (Table). Analysing patients who were positive at baseline for each of the isotypes, we observed that $19 / 48(40 \%), 16 / 43(37 \%)$ and $11 / 48(23 \%)$ of the patients positive for the IgG isotype became negative on ABA+MTX, ABA and MTX, respectively, at 1 year. For the IgM isotype, 26/48 (54\%), 14/36 (39\%) and $15 / 38(39 \%)$ became negative on ABA+MTX, ABA and MTX, respectively. For the IgA isotype, $12 / 26(46 \%), 10 / 23(43 \%)$ and $13 / 31$ (42\%) became negative on ABA+MTX, ABA and MTX, respectively.

Conclusions: Concentrations of all anti-CarP isotypes (IgM, $\lg A, \lg G$ ) were numerically reduced by abatacept+MTX therapy compared with MTX or abatacept alone. Abatacept+MTX trended towards higher rates of seroconversion to negative for all isotypes over 1 year of treatment. These results indicate that the extent of the anti-CarP antibody response can be modulated by intervention with abatacept on background MTX in anti-citrullinated protein antibody-positive patients with early RA.

References:

[1] Emery P, et al. Ann Rheum Dis 2015;74:19-26.

Disclosure of Interest: L. Trouw: None declared, S. Connolly Shareholder of: Bristol-Myers Squibb, Employee of: Bristol-Myers Squibb, A. Johnsen Employee of: Bristol-Myers Squibb, J. Ye Shareholder of: Bristol-Myers Squibb, Employee of: Bristol-Myers Squibb, M. Maldonado Shareholder of: Bristol-Myers Squibb, Employee of: Bristol-Myers Squibb, R. Toes: None declared, T. Huizinga
Grant/research support from: EU \& Dutch Arthritis Foundation, Consultant for: Abbott Laboratories, Biotest AG, Bristol-Myers Squibb, Crescendo Bioscience, Inc, Novartis Pharmaceuticals Corporation, Pfizer Inc, Roche, sanofi-aventis, Schering-Plough, UCB, Inc., Eli Lilly, Speakers bureau: Abbott Laboratories, Biotest AG, Bristol-Myers Squibb, Novartis Pharmaceuticals Corporation, Pfizer Inc, Roche, sanofi-aventis, Schering-Plough

DOI: 10.1136/annrheumdis-2017-eular.2935

\section{AB0249 IDENTIFICATION OF INFLAMMATORY- AND IMMUNE DISORDER- RELATED PROTEINS AS PUTATIVE BIOMARKER CANDIDATES FOR IMPROVING RHEUMATOID ARTHRITIS MONITORING}

L. González-Rodríguez ${ }^{1}$, V. Calamia ${ }^{1}$, L. Lourido ${ }^{1}$, P. Fernández-Puente ${ }^{1}$, M. Camacho-Encina ${ }^{1}$, C. Ruiz-Romero ${ }^{1}$, A. Julià ${ }^{2}$, A. Fernández-Nebro ${ }^{3}$, J. Tornero ${ }^{4}$, S. Marsal ${ }^{2}$, F.J. Blanco ${ }^{1} .{ }^{1}$ Proteomics Group, Rheumatology Division, ProteoRed, PRB2-ISCIII, INIBIC-Hospital Universitario A Coruña, A Coruña; ${ }^{2}$ Rheumatology Research Group, Vall d'Hebron Hospital Research Institute, Barcelona; ${ }^{3}$ UGC Reumatología, Instituto de Investigación Biomédica (IBIMA), Hospital Regional Universitario de Málaga, Málaga; ${ }^{4}$ Hospital Universitario Guadalajara, Guadalajara, Spain

Background: Rheumatoid arthritis (RA) is a long-lasting inflammatory autoimmune disorder that ultimately leads to the destruction of joint architecture. Objectives: Using the DAS28 activity index, 80 RA serum samples (40 with low activity and 40 with high activity) were selected in order to be analyzed by mass spectrometry. The aim of this study was to find possible protein biomarkers that could discriminate patients with different RA activity in the daily clinical routine. Methods: In order to facilitate the complex measurement of these serum samples, a simple, fast and reproducible albumin-specific depletion method using ethanol was optimized and applied to this study. Four independent pools of the 40 high RA activity samples (10 samples per pool) and 4 pools of the 40 low RA activity samples were firstly albumin-depleted, and then the remnant serum proteins were digested and differentially labelled with iTRAQ 8-plex reagents. Subsequently, the 8 labelled pools were combined and cleaned using StageTips-C18. Finally, the pool mixture was fractionated by HPLC (Zorbax-C18) and the resulting fractions were analyzed by nanoLC-MS/MS using two different equipments for validation (MALDI-TOF/TOF and TripleTOF).

Results: The mass spectrometry analysis led to the identification of 186 proteins. Among these, Haptoglobin, Kininogen-1, Alpha-2-HS-glycoprotein, Serum Amyloid A, Afamin and Histidine-rich-glycoprotein, exhibited a differential relative abundance depending on the RA activity of the patients $(p<0.03)$ in both analysis. These proteins were also validated by other orthogonal techniques (western blot, ELISA and protein arrays).

Conclusions: In this proteomic study, 9 proteins were found to be modulated between patients with high and low RA activity. Most of these proteins are related with the RA process and the effects caused by this type of disease (inflammation and immune disorder in joints). Therefore, these proteins are possible biomarker candidates for improving RA monitoring. Future validation experiments and prospective studies are needed to facilitate their implementation in the clinical routine.

Disclosure of Interest: None declared

DOI: 10.1136/annrheumdis-2017-eular.4912

\section{AB0250 THE ADIPOSE TISSUE AS PREDICTIVE FACTOR OF DISEASE ACTIVITY IN RHEUMATOID ARTHRITIS PATIENTS: EVALUATION OF BODY FAT COMPOSITION BY BIOELECTRICAL IMPEDANCE ANALYSIS AND ULTRASONOGRAPHY}

M.G. Anelli ${ }^{1}$, C. Rotondo ${ }^{2}$, R. Fanizzi ${ }^{2}$, O. Magazzino ${ }^{2}$, M. Giannotta ${ }^{2}$, M. Giannini $^{2}$, O. Lorusso $^{3}$, E. Praino ${ }^{2}$, M.T. Viggiani ${ }^{3}$, F. Cacciapaglia ${ }^{2}$ M. Barone ${ }^{3}, \mathrm{G}$. Lapadula ${ }^{2}$, F. Iannone ${ }^{2} \cdot{ }^{1}$ DIM; ${ }^{2}$ DETO, Unit of Rheumatology; ${ }^{3}$ DETO, Gastroenterology Unit, Bari, Italy

Background: Adipose tissue (AT) is an endocrine organ able to secrete the "adipokine" molecules that contribute to the low-grade inflammatory state in obese subjects and to the local inflammation that affects joints and bone $(1,2)$. High-grade inflammation, in course of RA, leads to an altered body composition $(3,4)$, characterized by the increasing of fat mass and the decreasing of lean mass, mostly not associated to body mass index (BMI) variations $(3,5)$. The BMI is not able to differentiate visceral (VTH) and subcutaneous (STH) fat tissue and to distinguish between muscle mass and fat mass body composition (BC) $(6,7)$. Alternative methods proposed for assessment of visceral fat deposition, are bioelectrical impedance analysis (BIA) and ultrasonography (US).

Objectives: The aim of the study is to investigate if BC, assessed by BIA and US,

Abstract AB0248 - Table 1. Median \% change from baseline (25\%, $75 \%$ ) for anti-CarP isotypes

\begin{tabular}{|c|c|c|c|c|c|c|}
\hline & \multicolumn{3}{|c|}{ Day 85} & \multicolumn{3}{|c|}{ Day 365} \\
\hline & $\lg G$ & $\lg M$ & $\lg A$ & $\lg G$ & $\lg M$ & $\lg A$ \\
\hline$\overline{\mathrm{ABA}}$ & $-17.3(-55.7,0.0)$ & $-26.3(-57.9,0.0)$ & $-6.8(-35.1,0.0)$ & $-31.2(-67.4,0.0)$ & $26.0(-81.2,0.0)$ & $-26.7(-72.9,13.0)$ \\
\hline MTX & $-19.3(-53.6,0.0)$ & $-35.7(-54.4,-6.9)$ & $-27.2(-42.4,-3.9)$ & $-17.7(-65.1,0.0)$ & $-38.3(-63.7,0.0)$ & $-21.9(-50.3,0.0)$ \\
\hline $\mathrm{ABA}+\mathrm{MTX}$ & $-38.8(-62.3,0.0)$ & $-44.2(-59.5,-13.8)$ & $-41.3(-54.9,-28.3)$ & $-55.7(-76.7,0.0)$ & $-45.7(-72.5,-0.2)$ & $-46.4(-66.7,0.0)$ \\
\hline
\end{tabular}


correlates with disease activity (assessed by the Disease Activity Score using 28 joint counts - DAS28) and affects the response to the therapy (DAS 28 variation from first evaluation).

Methods: 87 consecutive RA patients (pts) (72 women and 15 men; aged $52.4 \pm 13.2$ years; disease duration of $10.7 \pm 8.6$ years), treated with DMARDs and/or biologics (bDMARDs), were recruited during their regular visit. The inclusion criteria were the 1987 American College of Rheumatology (ACR) or ACR/EULAR 2010 classification criteria. The pts underwent to anthropometric measures (BMI); abdominal US to assess STH and VTH and derived computing of peritoneal circumference (PC); and BIA to the indices of body composition (fat-free mass index (FFMI) and fat mass index (FMI).

Results: We observed increasing values of BMI, FMI, VTH (fig. 1) and CP with the worsening of disease activity phases, evaluated by DAS 28. In particular, pts with DAS28 $\geq 5.1$ had highest BMI $(30,9 \pm 2 ; p=0,036)$, FMI $(11,5 \pm 1,6 ; p=0,05), C P$ $(92,7 \pm 12,5 \mathrm{~cm} ; \mathrm{p}=0,035)$ and VTH $(24,8 \pm 5,8 \mathrm{~mm} ; \mathrm{p}=0,046)$ than pts in less severe disease activity. By linear regression analysis the predictor of higher DAS28 is the BMI $(p=0,028)$. As regard the drug response, the predictors of DAS 28 improvement are higher FFMI $(p=0,044)$ and lower $B M I(p=0,015)$, independently by bDMARDs or DMARDs treatment. A trend to higher FMI and US AT measures was observed in female with high disease activity, in particular in menopause pts.

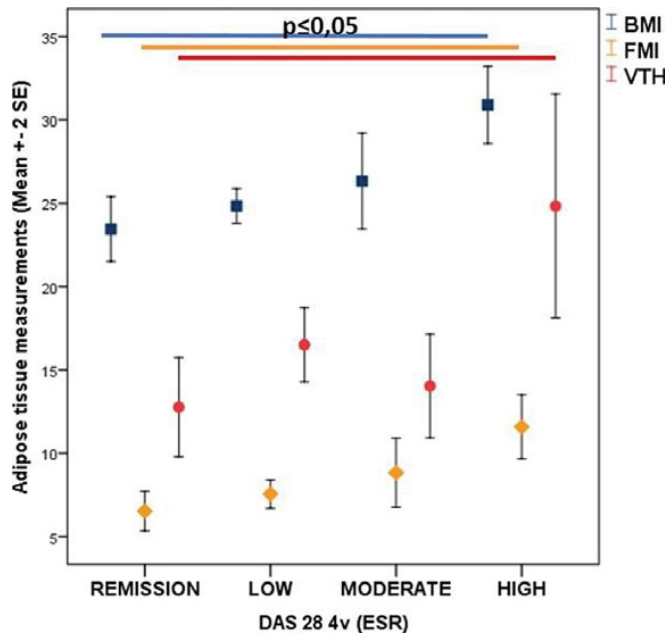

Conclusions: An altered fat distribution is observed in active RA phases; in particular, the FMI increasing is attributable just to visceral AT (VTH and CP). An inflammatory hyperactivity of visceral adiposity could be supposed in RA. The body composition, in addition to BMI, seems to predict the disease activity and drug response in RA patients. The evaluation of VTH by US could be useful to not overestimate the disease activity; instead the BIA could be a useful tool to support the clinicians in a more aggressive treatment management.

References:

[1] Nat Clin Pract Rheumatol 2007; 3(12):716-24.

[2] J Mol Endocrinol 2009; 43(1):11-8.

[3] Mediators Inflamm 2013; 2013:710928.

[4] Arthritis Care Res (Hoboken) 2012; 64(10):1471-9.

[5] Nat Rev Rheumatol 2010; 6(8):445-51.

[6] Curr Opin Clin Nutr Metab Care 2003; 6(4):387-93.

[7] Ann Rheum Dis 2007; 66(10):1316-21.

Disclosure of Interest: None declared

DOI: 10.1136/annrheumdis-2017-eular.4260

\section{AB0251 IN RHEUMATOID ARTHRITIS EROSIONS ARE MORE FREQUENT IN THE FEET THAN IN THE HANDS}

M.L. Andersson, B. Svensson, K. Forslind. Department of Clinical Sciences, Section of Rheumatology, Lund University, Lund and Helsingborg, Sweden

Background: Joint destruction in patients with rheumatoid arthritis (RA) is mostly evaluated by scoring radiographs of both hands and feet using the Sharp van der Heijde or Larsen scoring methods. In contrast, DAS28, the most commonly used composite measure of inflammation measure, does not include the feet.

Objectives: To study the distribution of erosions in hand and wrist (hands) and feet in early RA and elucidate if the feet should be included also in composite measures of inflammation

Methods: This study comprises 1052 patients from the BARFOT study of patients with early RA, recruited 1992-2006. Radiographs of hands and feet were performed at baseline, 1, 2, 5 and 8 years and evaluated by the Sharp van der Heijde scoring (SHS) method (32 joints in the hands and 12 in the feet). Disease activity at baseline was measured by the DAS28.

Results: In the feet there were significantly more eroded joints in percent of examined joints at all time points $(p<0.001)$, and higher erosion scores in percent of maximum erosion score at 5 and 8 years $(0.037$ and 0.021 respectively), compared with the hands.
There were no differences in mean joint space narrowing (JSN) between hands and feet at any time point. Patients with erosions only in the feet had significantly lower DAS28, mean 4.59, compared with the patients in the other groups, mean 5.03 in the no erosions group $\mathrm{p}=0.031$, mean 5.17 in the group with erosions only in the hands, $p=0.013$, and mean 5.15 in the group with erosions in both hands and feet, $p=0.031$.

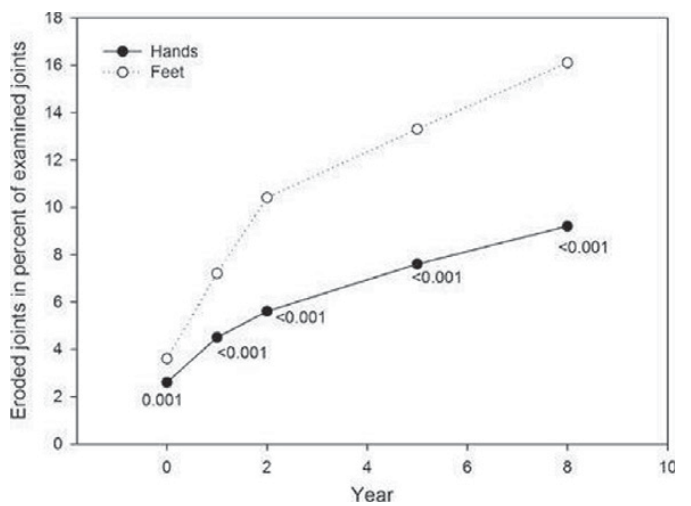

Conclusions: Joint destruction over time was more pronounced in the feet than in the hands. Baseline erosions limited to the feet were associated with low disease activity, suggesting that inflammation localized to the feet may not be reflected by DAS28. These observations may have relevance to the evaluating of disease activity and progression in the individual patient. Possibly inclusion of the feet to DAS28 might improve the validity of this disease activity measure.

Disclosure of Interest: None declared

DOI: 10.1136/annrheumdis-2017-eular.3634

\section{AB0252 THE IMPACT OF THE RHEUMATOID FOOT ON FUNCTION IN PATIENTS WITH RHEUMATOID ARTHRITIS EVALUATED BY FFI AND LFIS}

M. Erraoui, B. Amine, L. Tahiri, I. El Binoune, J. Bahha, S. Fellous, Y. Boujenane F. Allali, R. Bahiri. Rheumatology, Mohammed V University, Faculty of Medicine and Pharmacy of Rabat, El Ayachi Hospital, SALE, Morocco

Background: The impairment of function in patients with rheumatoid arthritis (RA) is determined by several factors related to the disease including joint damage. The foot, a location frequently affected during course of the disease, has a major impact on the lower limb and could cause functional disability.

Objectives: The purpose of this study is to evaluate the impact of the rheumatoid foot on function of patients with RA.

Methods: Cross-sectional study was conducted in 33 patients with RA. Patients with static lower limb disorder or foot injury from other origin were excluded. Demographic and clinical characteristics were collected: age, sex, BMI, disease duration, tender joint count, swollen joint count, foot pain evaluated on an VAS, foot squeeze test and various podiatric abnormalities observed clinically (forefoot, midfoot and rearfoot). Biological characteristics also collected: sedimentation rate in the first hour, $C$ reactive protein, rheumatoid factor and anti-CCP. Disease activity was evaluated by DAS28, CDAI, SDAI and DAS44. Functional repercussions were estimated by the French Functional Index (FFI), comprising 23 items, divided into 3 sections: pain, function and limitation of activity. Functional disability was studied by the Leeds Foot Impact Scale (LFIS), which includes 51 items (21 items specific to foot function alteration (LFIS-I) and 30 related to foot disability (LFIS-D)). Statistical analysis was performed using SPSS21 software.

Results: Thirty-three patients followed for RA were included. The mean age of our patients was $49.39 \pm 10.52$ with a female predominance $(87.9 \%)$. Mean disease duration was $9.96 \pm 7.49$ years. In all patients; 21 (95.5\%) were seropositive. The mean DAS28 was $5.53 \pm 1.38$ and the mean $\mathrm{HAQ}$ was $1.37 \pm 0.83 .93 .9 \%$ of our patients had bilateral foot pain; $69.7 \%$ in the forefoot, $18.2 \%$ in the midfoot and $42.4 \%$ in the hindfoot. The medial retromalleolar tumefaction was found in $21.2 \%$ of the patients and the lateral retromalleolar tumefaction in $45.5 \%$ of them. The squeeze test was positive in $23(69.7 \%)$ patients. Prevalences of Podiatric abnormalities were noted in the following ordre: hallux valgus $(48.5 \%)$, quintus varus $(12.1 \%)$, hallux rigidus $(6.1 \%)$, claw toe $(15.2 \%)$, triangular forefoot $(9.1 \%)$, rearfoot valgus $(27.3 \%)$ and rearfoot varus $(27.3 \%)$.

Mean FFI was $52.35 \pm 25.63$ (FFI-pain: $58.69 \pm 24.41$, FFI-function: $53.66 \pm 30.48$ and FFI- limitation of activity: $39.33 \pm 30.58$ ). Mean LFIS-I was $11.48 \pm 5.36$ and mean LFIS-D was 19.96 .

A high $\mathrm{FFI}$ was associated with foot pain $(p=0.034)$; in midfoot $(p=0.029)$ and rearfoot $(p=0.005)$ and with high disease activity: DAS28 $(P=0.005)$, CDAI, SDAI and DAS44 $(p=0.0001)$

LFIS-I and LFIS-D were statistically related to pain in foot $(\mathrm{p}=0.019)$ (midfoot $(0.042)$, hindfoot $(0.003)$ ), and to a positive squeeze test. The disease activity was positively correlated with high LFIS-I and LFIS-D $(p=0.0001)$. The presence of medial retro-malleolar swelling, posterior gutter filling and calcaneal varus is responsible for a high FFI, LFIS-I and LFIS-D with respectively: $p=0.0001$, $\mathrm{p}=0.003, \mathrm{p}=0.003$. 